Research Paper

\title{
Free CA125 promotes ovarian cancer cell migration and tumor metastasis by binding Mesothelin to reduce DKK1 expression and activate the SGK3/FOXO3 pathway
}

\author{
Qianyu Huo ${ }^{1 \#}$, Chen $\mathrm{Xu}^{2 \#}$, Yanhong Shao ${ }^{1}$, Qin $\mathrm{Yu}^{1}$, Lunhui Huang ${ }^{1}$, Yunde $\mathrm{Liu}^{1 凶}$ and Huijing Bao ${ }^{3 凶}$ \\ 1. School of Medical Technology, Tianjin Medical University, Tianjin 300203, China. \\ 2. Laboratory Science Department, Tianjin 4th Central Hospital, Tianjin, 300100, China. \\ 3. Integrative Medical Diagnosis Laboratory, Tianjin Nankai Hospital, Tianjin, 300100, China; School of Medical Technology, Tianjin Medical University, Tianjin 300203, \\ China. \\ \#Contributed equally to this work \\ $\triangle$ Corresponding authors: E-mail: lyd@tmu.edu.cn (Y Liu); kris_10713@126.com (H. Bao).
}

(C) The author(s). This is an open access article distributed under the terms of the Creative Commons Attribution License (https://creativecommons.org/licenses/by/4.0/). See http://ivyspring.com/terms for full terms and conditions.

Received: 2020.08.20; Accepted: 2020.12.18; Published: 2021.01.14

\begin{abstract}
Objective: CA125/MUC16 is an O-glycosylated protein that is expressed on the surfaces of ovarian epithelial cells. This molecule is a widely used tumor-associated marker for diagnosis of ovarian cancer. Recently, CA125 was shown to be involved in ovarian cancer metastasis. The purpose of this study was to investigate the mechanism of CA125 during ovarian cancer metastasis.

Methods: We analyzed the Oncomine and CSIOVDB databases to determine the expression levels of DKK1 in ovarian cancer. DKK1 expression levels were upregulated or downregulated and applied with CA125 to Transwell and Western blot assays to ascertain the underlying mechanism by which CA125 stimulates cell migration via the SGK3/FOXO3 pathway. Anti-mesothelin antibodies (anti-MSLN) were used to block CA125 stimulation. Then the expression levels of DKKI were tested by enzyme-linked immunosorbent assay (ELISA) to eliminate the blocking effect of anti-MSLN to CA125 stimulation. Xenograft mouse models were used to detect the effects of CA125 and anti-MSLN on ovarian cancer metastasis in vivo.

Results: DKK1 levels were downregulated in ovarian tumor tissues according to the analyses of two databases and significantly correlated with FIGO stage, grade and disease-free survival in ovarian cancer patients. DKK1 levels were downregulated by CA125 stimulation in vitro. Overexpression of DKK1 reversed the ability of exogenous CA125 to mediate cell migration by activating the SGK3/FOXO3 signaling pathway. Anti-MSLN abrogated the DKK1 reduction and increased the apoptosis of ovarian cancer cells. The use of anti-MSLN in xenograft mouse models significantly reduced tumor growth and metastasis accelerated by CA 125 .

Conclusions: These experiments revealed that the SGK3/FOXO3 pathway was activated, wherein decreased expression of DKK1 was caused by CA125, which fuels ovarian cancer cell migration. Mesothelin is a potential therapeutic target for the treatment of ovarian cancer metastasis.
\end{abstract}

Key words: CA125, DKK1, MSLN, therapy, ovarian cancer

\section{Introduction}

Ovarian cancer (OC) is a highly aggressive tumor and the most fatal of all common gynecological malignancies in women worldwide [1]. One of the main reasons for the high mortality is that patients cannot be diagnosed before an advanced stage. The 5 -year survival among women diagnosed with OC is $46.2 \%$. Over $70 \%$ of OC is not diagnosed before progressing to stage III or IV [2]. In epithelial ovarian cancer, late-stage disease has a 5-year relative survival rate of $29 \%$, in contrast to $92 \%$ for early-stage disease [3]. Tumors likely invade adjacent organs or metastasize to the peritoneal cavity at an early stage in progression [4]. According to the staging classification of ovarian cancer, extension or metastasis to extraovarian/extratubal pelvic organs is defined as stage II, metastasis to the retroperitoneal lymph nodes is defined as stage III, and distant metastasis is defined as stage IV [5]. Thus, in ovarian cancer, tumor 
invasion and metastasis play a decisive role in the progression of disease.

An abnormal level of CA125 was observed in 99\% of serous carcinoma cases rated I to IV in FIGO staging [6]. CA125 (MUC16) is currently a well-established serum tumor marker for ovarian epithelial cancer and may diagnose cancer at an early stage $[7,8]$. Only 1 percent of healthy persons and 6 percent of patients with nonmalignant disease had serum CA125 levels above $35 \mathrm{U}$ per milliliter. However, 82 percent of surgically demonstrated ovarian cancer patients had elevated levels of CA125, and among those, levels of CA125 correlated with progression or regression of disease in 93 percent of the cases [9]. In postoperative patients who underwent cytoreductive or radical surgery and chemotherapy, serum CA125 values could indicate a relative risk of recurrence [10,11]. The glycoprotein carrying the CA125 antigen is encoded by the mucin 16 gene in humans [12]. Mucins (MUCs) are large and heavily glycosylated proteins with a high carbohydrate content and are found at the cell surface of many epitheliums [13,14]. MUC16 is a transmembrane type of mucin that is expressed on the cell surface and provides both barrier and signaling functions [15]. Soluble proteolytic fragments named CA125 are released into the extracellular space and detected in the serum. CA125 has a relatively high concentration throughout the process of OC [16]. Recently, an increasing number of studies have described the biological role of MUC16 in the progression and metastasis of ovarian tumors [17]. The expression of MUC16 increases ovarian cancer cell motility, invasiveness, and metastatic properties and enhances tumor growth and metastases of ovarian cancer in SCID mice [18].

In our previous study, we examined whether CA125 promotes ovarian cancer cell migration and whether DKK1 reverses the ability of CA125 to induced migration. We also speculated that the serum CA125 concentration could serve as an effective index of metastasis in ovarian cancer [19]. The present study was designed to determine the possible mechanism of CA125 in the metastasis of ovarian cancer. CA125 enhanced cell migration by reducing DKK1 expression and activating the SGK3/FOXO3 signaling pathway. Furthermore, by blocking the binding of free CA125 and mesothelin (MSLN) on the cell membrane, the decrease in DKK1 can be reversed. Applying a mouse monoclonal antibody targeting MSLN in mice bearing ovarian cancer cell xenografts significantly suppressed tumor growth and tumor lymphatic drainage. Our results demonstrated that CA125 via the MSLN/DKK1/SGK3/FOXO3 pathway accelerates cell migration, and targeting mesothelin may potentially be utilized in ovarian cancer therapy.

\section{Materials and methods}

\section{Gene expression and survival analysis in databases}

CSIOVDB (http://csiovdb.mc.ntu.edu.tw/ CSIOVDB.html) is a database established for the study of ovarian cancers, including transcriptomic microarray data from 3,431 human ovarian cancer tissues with clinicopathological parameters [20]. Here, the database was used to investigate the expression of DKK1 in ovarian cancer tissues and its correlation with pathological grade and clinical outcome.

DKK1 mRNA expression in ovarian cancer was investigated in the Oncomine Cancer Profiling Database (https://www.oncomine.org/) (Compendia Bioscience, Ann Arbor, MI). The filter was set as follows: gene as DKK1; analysis type as ovarian cancer vs. normal; data type as mRNA. The results are presented as box plots.

\section{Cell lines, antibodies and other reagents}

A2780, CaOV-3, OVCAR3, and skov-3 cell lines were purchased from the American Type Culture Collection (ATCC). A2780, CaOV-3, and skov-3 cell lines were maintained in RPMI 1640 medium (Gibco, Invitrogen, CA, USA) supplemented with $10 \%$ fetal bovine serum (FBS) (Gibco, Invitrogen, CA, USA). The OVCAR3 cell line was maintained in RPMI 1640 medium supplemented with $20 \%$ FBS and $1 \%$ bovine insulin (Sigma-Aldrich, St. Louis, MO, USA). Cells were incubated at $37^{\circ} \mathrm{C}$ in a humidified incubator containing 5\% $\mathrm{CO}_{2}$ in air. Rabbit anti-Akt (1:1000) (\#92725), rabbit anti-p-Akt (1:1000) (\#4060), and rabbit anti-PI3K (1:500) (\#4292) antibodies were all purchased from Cell Signaling Technology (Danvers, MA, USA). Rabbit anti-SGK3 (1:1000) (bs-6475R) antibody was purchased from Bioss (Beijing, China). Rabbit anti-FOXO3A (1:2000) (ab12162) and Rabbit anti-LYVE-1 (1:300) (ab 14917) was purchased from Abcam (Cambridge, UK). Anti-GAPDH (1:2000) (AF7021) and anti-Tubulin- $\beta$ (1:2000) (T0023) antibodies were purchased from Affinity Biosciences (Jiangsu, China). Mouse-anti-rabbit immunoglobulin G (sc-2357) (1:1000), mouse IgGK binding protein-HRP (1:1000) (sc-516102) and mouse anti-mesothelin (1:50) (sc-365324) antibodies were purchased from Santa Cruz Biotechnology, Inc (Texas, USA). ECL Western Blotting Detection Reagents were obtained from Pierce (Texas, USA). Lipo 2000 transfection reagent was purchased from Thermo Fisher Scientific (CA, USA). Recombinant human CA125/MUC16 was purchased from R\&D Systems (Minneapolis, MN). 


\section{Treatment with CA125 and anti-MSLN antibody}

Ovarian cancer cells were incubated with $0,0.2$, and $0.4 \mu \mathrm{g} / \mathrm{mL}$ recombinant human CA125 for $48 \mathrm{~h}$ as previously described [19]. For further blocking assays, cells were treated with anti-MSLN monoclonal antibody at a dilution ratio of 1:50 $(4 \mu \mathrm{g} / \mathrm{mL})$. Two days later, the cells treated with the corresponding treatments were washed with phosphate buffered saline (PBS) or harvested for assays accordingly.

\section{RNA extraction, reverse transcription and quantitative PCR}

RNA was isolated from OC cell lines using TRIzol reagent (Ambion, USA) according to the manufacturer's instructions. Reverse transcription was performed using a FastQuant RT Kit (Tiangen Biotech, China). Quantitative PCR reactions were conducted with SYBR Green PCR Master Mix Reagents (Tiangen Biotech, China) on the Stratagene MX3005P system. The fold change $(2-\Delta \Delta \mathrm{Ct})$ in expression was calculated for the gene of interest relative to the internal control gene (GAPDH). The primer sequences used in PCRs were as follows: GAPDH: sense: 5'-CCT CCA AGG AGT AAG ACC CC-3', antisense: 5'-AGG GGT CTA CAT GGC AAC TG-3'. The primer sequences for DKK1 (Hs-QRP40337) were purchased from GeneCopoeia, China.

\section{Immunoblot analysis}

Cells were washed and lysed in loading buffer (KeyGEN BioTECH, China). Proteins were separated by ultrasonic and then resolved by SDS-PAGE, transferred to polyvinylidene fluoride (PVDF, 0.45 $\mu \mathrm{m}$, Millipore, Germany) membranes and detected using specific primary antibodies, appropriate HRP-conjugated secondary antibodies and an ECL detection system. The nuclear/cytoplasmic extracts were prepared using the nuclear and cytoplasmic protein extraction kit (P0028, Beyotime, China) as per the manufacturer's instructions. Next, ImageJ software was used for densitometric analysis of the bands.

\section{Transient transfection}

The DKK1 DNA fragments were cloned into the pEGFP-N1 plasmid (Tongyong, China). The plasmids were extracted from Escherichia coli DH5a transformed cultures using Endotoxin free Plasmid Extraction Kit (DP117, Tiangen Biotech, China) as per the manufacturer's instructions. The pEGFP-N1and pEGFP-N1-DKK1 plasmids $(2.5 \mu \mathrm{g} / \mathrm{mL})$ were transfected into logarithmic growth phase ovarian cancer cells using Lipofectamine 2000 (Invitrogen, CA, USA).
For knockdown of DKK1 expression, siRNA (GenePharma, China) was used in the ovarian cancer cell line. Cells were seeded into the plate, cultured for $24 \mathrm{~h}$ and transfected with $75 \mathrm{pM}$ negative control (NC) RNA or siRNA using Lipofectamine 2000 according to the manufacturer's instructions. The siRNA sequences were sense: 5'- GCCGGAUACAGAAAGAUCATT-3' and antisense: 5'- UGAUCUUUCUGUAUCCGGCTT -3 '.

After $24 \mathrm{~h}$ transfection, Western blots, qPCR, ELISAs and further experiments were conducted.

\section{ELISA}

Cell culture medium was pipetted into tube and centrifugated for achieving supernatant. Determination of DKK1 in culture medium was performed using a commercial ELISA kit (EK0867) (Boster, China) according to the manufacturer's instructions. The measured intensity of the developing color was proportional to the concentration of DKK1. The O.D. absorbance was read with a microplate reader (Synergy2, USA) at 450 $\mathrm{nm}$. The results are presented as O.D. levels.

\section{Transwell assays}

The cells that were transiently transfected were harvested and seeded in transwell inserts (polycarbonate filter with $8 \mu \mathrm{m}$ pores, Costar, USA). After overnight incubation, medium containing different concentrations of CA125 was added to observe the changes in cell migration. After $48 \mathrm{~h}$ of culture at $37^{\circ} \mathrm{C}$, cells that remained inside the upper chamber were removed softly with a cotton swap. Cells that migrated to the lower surface of the filter membrane were fixed with anhydrous methanol and stained with $0.1 \%$ crystal violet. Filters were air dried, and photos were taken with an inverted phase contrast microscope (Nikon Eclipse Ti-U, Japan). The invading cells in 4 fields were counted, and statistical analysis was performed.

\section{Transcriptome sequencing analyses of CA125-treated OC cells}

A total of $5 \times 10^{5}$ A2780 cells were seeded per well in 6-well plates. After overnight incubation, the culture medium was changed to medium with or without CA125 $(0 \mu \mathrm{g} / \mathrm{mL}$ or $0.2 \mu \mathrm{g} / \mathrm{mL})$. The cells were incubated for $48 \mathrm{~h}$, harvested in TRI-Zol and reverse transcribed. cDNA libraries were transcriptome sequenced on an Illumina HiSeq platform with paired-end 150 bp read length. Screening of differentially expressed genes was based on Poisson distribution methods. Differentially expressed genes were used for subsequent pathway analysis. 


\section{Apoptosis detection and flow cytometry}

The percent of cells that underwent apoptosis was detected by using the FITC Annexin-V and PI detection kit (KGA107, KeyGEN BioTECH, China). The procedure was performed according to the manufacturer's instructions. Briefly, the cells (approximately $1 \times 10^{5}$ cells per tube) were harvested and washed and suspended in binding buffer provided by the manufacturer containing $5 \mu \mathrm{L}$ of FITC Annexin-V and $5 \mu \mathrm{L}$ of PI for 5 to $15 \mathrm{~min}$ at room temperature in the dark. The cells were then vortexed adequately and analyzed by FACS Verse (BD Biosciences, Franklin Lakes, NJ, USA) within $1 \mathrm{~h}$. The data were analyzed by FlowJo (De Novo Software, Glendale, CA, USA). Ten thousand events were analyzed in each run.

\section{In vivo xenograft models and histological analysis}

All animal experimental procedures were approved by the Animal Care and Welfare Committee of Tianjin Medical University. A2780 or OVCAR3 cell pellets were suspended in PBS and injected subcutaneously on right back into NOD-SCID-IL2rg mice (6-8 weeks old, female) with $5 \times 10^{6}$ cells per mouse. In the experiment to verify the effect of CA125 on metastasis, the mice were injected with PBS, CA125 ( $0.2 \mu \mathrm{g}$ per mouse) via the tail vein after the tumors were palpable. The mice were sacrificed after three injections when the tumors were approaching the scope of ethical approval. In the treatment experiment, the mice were injected with PBS, CA125 ( $0.2 \mu \mathrm{g}$ per mouse) three times follow the cell seeded. Once the tumors were palpable, anti-mesothelin antibody ( $2 \mathrm{mg}$ per mouse) were injected four times via the tail vein in the mice which injected CA125 before.

Tumors were measured with calipers every two days in two directions, and the tumor volume was calculated using the formula (width ${ }^{2} \times$ length) $/ 2$. Evan's blue was injected around the tumor $2 \mathrm{~h}$ before the mice were sacrificed. After the animals were sacrificed, the tissues were separated. The tumors, axillary lymphatic tissues, and inguinal lymphatic tissues were fixed in $4 \%$ paraformaldehyde, embedded in paraffin and sectioned for hematoxylin and eosin (H\&E) staining and immunohistochemistry (IHC) analysis.

\section{Statistics}

Statistical analyses were performed using GraphPad Prism 6.0 Software. Student's t-test was used to compare the differences between two groups. One-way ANOVA was used to compare the differences among more than two groups. The results are presented as the mean $\pm S D$. A $p$ value of less than 0.05 was considered statistically significant.

\section{Results \\ DKK1 is downregulated in ovarian cancer tissue}

Aberrant expression of DKK1 (Dickkopf-1) has been observed in numerous human cancers. To investigate the role of DKK1 in ovarian cancer development, we determined the DKK1 expression level in ovarian cancer tissue versus normal tissue in the CSIOVDB (Figure 1) and Oncomine (Figure S1) databases. Generally, DKK1 expression levels were decreased in ovarian tumors compared with normal ovarian surface epithelium ( $p=1.6 \mathrm{E}-8)$ and decreased in tumor stroma compared with normal stoma $(p=3.8 \mathrm{E}-2)$ based on the CSIOVDB database (Figure 1A). DKK1 expression levels were also examined in different FIGO stages (Figure 1B) and FIGO grades (Figure 1C). Data indicated that compared with stage I patients, patients at advanced stage II $(p=8.62 \mathrm{E}-4)$, III $(p=6.76 \mathrm{E}-10)$ and IV $(p=5.6 \mathrm{E}-6)$ exhibited lower expression levels of DKK1. Similarly, compared with grade 1 tumors, tumors classified as poorly differentiated grade $2(p=6.22 \mathrm{E}-6)$ and $3(p=1.58 \mathrm{E}-10)$ had lower expression of DKK1. Furthermore, KaplanMeier survival analyses were performed using CSIOVDB. Among patients with lower DKK1 expression, poorer overall survival and disease-free survival were observed (Figure 1D). Different datasets of the Oncomine database are presented as box plots (Figure S1) and tables (Supplement Table 1). Briefly, lower expression of DKK1 was found in ovarian carcinoma and ovarian serous adenocarcinomas than normal tissue based on the Bonome and Yoshihara subdatasets.

\section{CA1 25 downregulated DKK1 expression in ovarian cancer cells}

The serum CA125 levels increased in most ovarian cancer patients. DKK1 decreased in ovarian cancer tissues at an advanced stage of disease progression. To determine the relationship between CA125 and DKK1 expression levels in ovarian cancer, we added CA125 to the culture medium of ovarian cancer cells. Since DKK1 is a secreted protein, the DKK1 mRNA and protein levels were detected by qPCR, Western blotting and ELISAs. The results demonstrated that the DKK1 mRNA levels were decreased with CA125 stimulation in A2780 and OVCAR3 cells (Figure 2A and B). Accordingly, ELISAs showed that the protein levels of DKK1 in the culture medium were decreased in two ovarian cancer cell lines (Figure 2C and D). However, the protein 
levels of DKK1 in OVCAR3 cells showed a decreased trend but no statistical significance. One potential reason is that OVCAR3 cells have higher expression levels of CA125, which may desensitize the cells to exogenous CA125. Western blot assays were conducted to examine the protein levels of DKK1 in the cell lysates, which showed nonsignificant changes (Figure 2E and F), indicating that the majority of DKK1 proteins were secreted into the culture medium.

\section{CA125 induced ovarian cancer cell migration by attenuating DKK1 expression}

We found that CA125 enhanced the migration of ovarian cancer cells [19]. CA125 also downregulated the expression of DKK1 in vitro. To address the relationship between the CA125-mediated decrease in DKK1 expression and the enhanced migration of cells, we transfected the pEGFP-N1-DKK1 vector and
siDKK1 into ovarian cancer cells to overexpress and knock down DKK1. The effects of DKK1 and CA125 on migration were observed in the Transwell assays. CA125 was observed to promote migration in both ovarian cancer cell lines. Figure $3 \mathrm{~A}$ shows that pretreatment with the OE DKK1 vector significantly blocked cell migration, which was enhanced by CA125. Figure 3B shows that siDKK1 synergistically enhanced CA125 efficacy to promote ovarian cancer cell migration. Overall, these data indicated that CA125, through attenuating DKK1 expression, modulates ovarian cancer cell migration.

\section{Next-generation sequencing of the differentially expressed transcripts of ovarian cancer cells with CA125 stimulation}

To explore the mechanisms by which CA125 enhances ovarian cancer cell migration, we conducted next-generation sequencing to investigate the

A

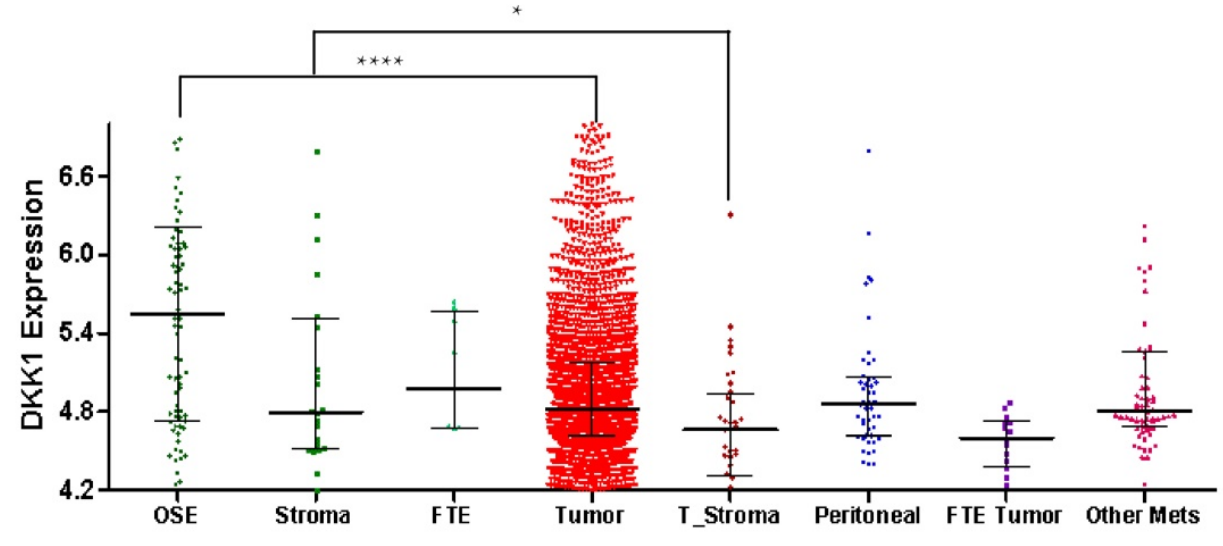

B

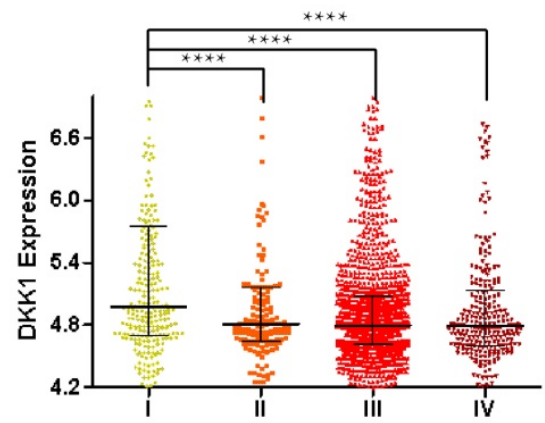

D

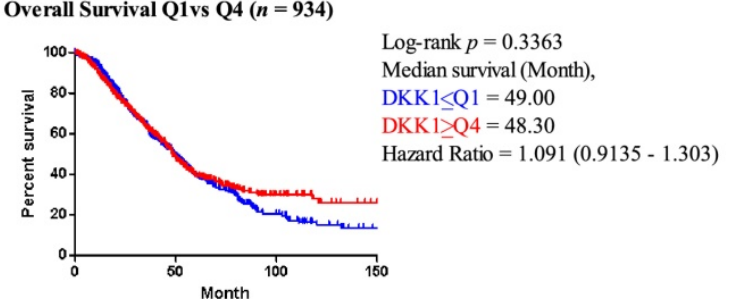

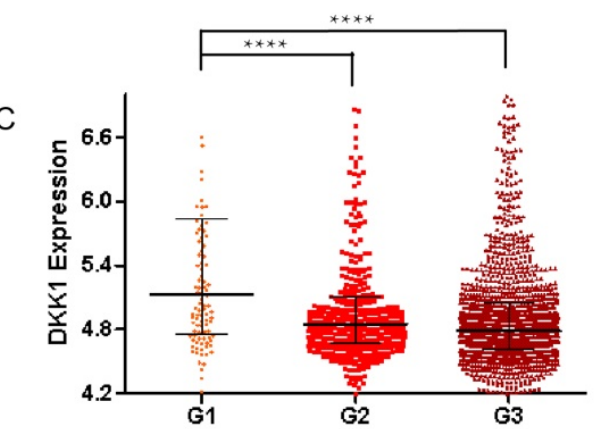

Disease-Free Survival Q1vs Q4 $(n=758)$

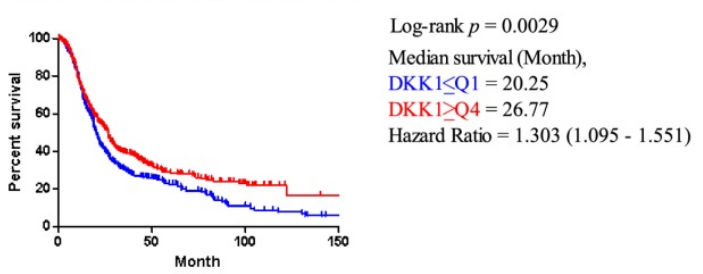

Figure 1. DKK1 expression levels in the CSIOVDB database. Gene expression profiles of DKK1 by the ovarian cancer disease state (A), FIGO stage (B), FIGO grade (C) and survival curve (D). Abbreviation: OSE, ovarian surface epithelium; FTE, fallopian tube epithelium; Mets, metastasis; Error bar is the median \pm quantile. Kaplan-Meier analyses were conducted to estimate overall survival and disease-free survival. $* \mathrm{p}<0.05$, $* * * * \mathrm{p}<0.0001$. 
differential expression of transcripts in the CA125-stimulated and unstimulated cells. HISAT (Hierarchical Indexing for Spliced Alignment of Transcripts) was used to map the clean reads to the reference genome. A total of 25526 transcripts were identified. Poisson distribution analysis was used to model the read counts. Twenty-one upregulated and twenty downregulated differentially expressed genes (DEGs) were identified with the threshold of absolute value of fold change $(\mathrm{FC})>1$ and false discovery rate (FDR) $\leq 0.001$ (Figure 4A-B). The expression patterns of the sample mRNAs are presented as heat maps (Figure 4C). Kyoto Encyclopedia of Genes and Genomes (KEGG) enrichment analysis was used to assess the DEGs affecting both the PI3K/Akt pathway and FOXO pathway (Figure 4D). SGK3 (serum/glucocorticoid regulated kinase family member 3) (fold change, 6.11; FDR, 8.6E-7) was upregulated and FOXO3 (forkhead box O3) (fold change, -7.47; FDR, 3.14E-24) was downregulated in the CA125-stimulated group compared with the unstimulated group. A schematic diagram of the pathway was acquired based on the KEGG database, and a hypothesis was proposed to explain the mechanism by which CA125 enhances cell migration (Figure 4E). Namely, CA125 promotes the migration of ovarian cancer cells by reducing DKK1 expression and activating the SGK3/FOXO3 pathway.

\section{CA125 reduced DKK1 expression to activate the SGK3/FOXO3 pathway}

SGKs (serum- and glucocorticoid-regulated kinases) are serine/threonine kinases that are similar to Akt; both phosphorylate $\mathrm{FOXO} 3$ and lead to the inhibition of FOXO3. FOXO3 primarily acts as a transcription factor in the nucleus, and phosphorylated FOXO3 binds to 14-3-3 protein and remains in the cytoplasm of cells [21]. To validate the SGK3 and FOXO3 expression levels following CA125 stimulation and the hypothesis proposed based on the sequencing results, we performed Western blot and semiquantitative analyses of the optical density of these proteins. Because PI3K is common upstream of SGK3 and Akt, we first detected the expression levels of Akt, p-Akt, PI3K, SGK3 and FOXO3. The Akt, p-Akt, PI3K, and SGK3 protein levels in total cells were not significantly changed; however, the content of FOXO3 in the nucleus had a decreasing trend. These results were partially consistent with the sequencing results (Figure 5A-B).

Table 1. Differentially expressed genes in pathway analysis

\begin{tabular}{ll}
\hline Pathway & Differentially expressed genes \\
\hline RNA transport & RGPD6; EIF3CL \\
Proteoglycans in cancer & LUZP6; MTPN \\
PI3K-Akt signaling pathway & SGK3; FOXO3B \\
Nucleotide excision repair & GTF2H2C; BIVM-ERCC5; BIVM \\
Lysine degradation & LUZP6; MTPN \\
FoxO signaling pathway & SGK3; FOXO3B \\
\hline
\end{tabular}
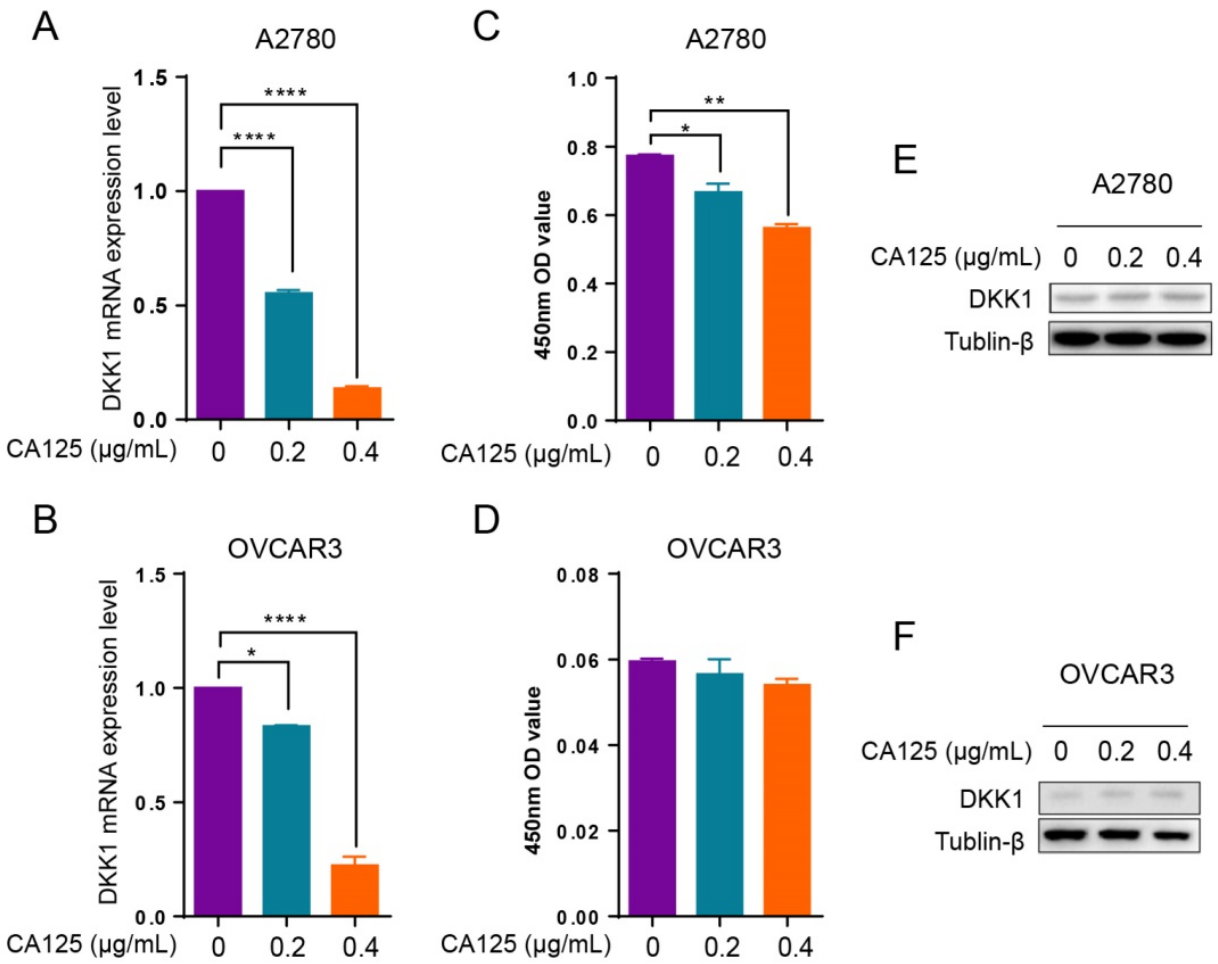

Figure 2. CA125 downregulates DKK1 expression in ovarian cancer cells. QPCR (A-B), ELISA (C-D), and Western blot (E-F) analysis of DKK1 mRNA levels in A2780 and OVCAR3 cells treated with CA125 at $0,0.2$. and $0.4 \mu \mathrm{g} / \mathrm{mL}$ for $48 \mathrm{~h}$. The results represent the mean $\pm \mathrm{SD}$. ${ }^{*} \mathrm{p}<0.05,{ }^{*} \mathrm{p}<0.01, * * * *{ }_{\mathrm{p}}<0.0001$. 


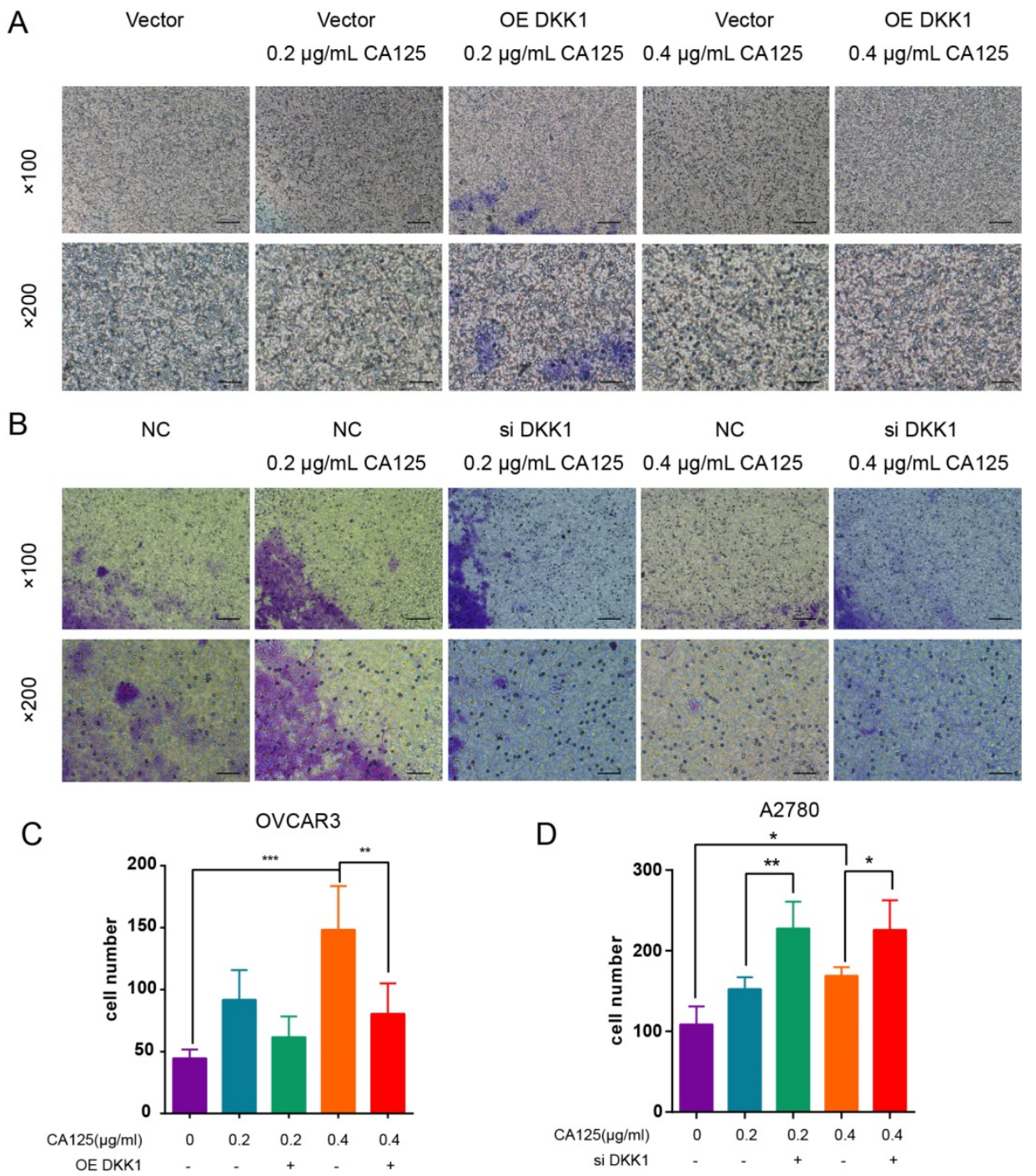

Figure 3. CA125 induced ovarian cancer cell migration by attenuating DKK1 expression. OVCAR3 cells (A) transfected with pEGFP-N1 vector and pEGFP-N1-DKK1 and A2780 (B) transfected with negative control (NC) and siDKK1 were subjected to Transwell migration assays and treated for $48 \mathrm{~h}$ with CA125 (scale bars $=100 \mu \mathrm{m}$ ). (C) (D) Cell counts across the Transwell membrane. The statistical analyses were conducted with the number of invaded cells (indicated by red arrows). The results represent the mean $\pm S D$. ${ }^{*} p<0.05, * * p<0.01, * * * p<0.001$.

Next, DKK1 was knocked down and overexpressed to determine the relationship between CA125-mediated reduction of DKK1 and activation of the SGK3/FOXO3 pathway. The expression of Akt, p-Akt, PI3K and SGK3 in the total cell lysates and the expression of FOXO3 in cytoplasmic/nuclear protein extracts were detected after transfection and stimulation with CA125 at $0,0.2$ and $0.4 \mu \mathrm{g} / \mathrm{mL}$. The activation of the SGK3/FOXO3 pathway was estimated by the expression of SGK3 and the translocation of FOXO3. The expression of SGK3 had an increasing trend, and FOXO3 translocated from the nucleus to the cytosol in siDKK1 cells synergistically with CA125 stimulation (Figure 5C-D). OE DKK1 reversed the effect of CA125 on activating the SGK3/FOXO3 pathway (Figure 5E-F). The relative expression levels of total SGK3 or nuclear FOXO3 were not significantly changed in the OE DKK1 cells with CA125 stimulation. Interestingly, the expression of Akt, p-Akt and PI3K in the siDKK1 cells was decreased after CA125 stimulation. One possible reason is that SGK3 and Akt share a large number of substrates, and activated downstream substrate feedback inhibits Akt, p-Akt and PI3K.

\section{Anti-MSLN blockade of CA1 25 reduced the decrease in DKK1 and initiated apoptosis}

MSLN is expressed on the surface of mesothelial cells, mesothelioma and ovarian cancer cells. To date, CA125 is the only known binding partner that interacts with MSLN [22]. We hypothesized that the effect of CA125 on ovarian cancer cells was induced by the binding of CA125 to MSLN. To block this effect, we added anti-MSLN and CA125 to the cell culture medium. DKK1 protein expression did not change $(p>0.05)$ in two ovarian cancer cell lines 
stimulated with CA125 and anti-MSLN compared to the unstimulated cell line (Figure 6A). Dramatically, the cells treated with anti-MSLN showed a decreasing tendency to proliferate. The expression levels of Tubulin- $\beta$ in total lysates were decreased by adding anti-MSLN and CA125 (Figure 6B). Subsequently, apoptosis was evaluated by Annexin/PI dual staining followed by flow cytometry to investigate the effect of CA125 and anti-MSLN. Cells were gated into four groups by staining according to the standard method [23]. Figure 6C-D shows that CA125 significantly reduced late apoptotic necrosis at high concentrations $(\mathrm{p}<0.05)$. However, with the addition of anti-MSLN, necrosis and late apoptotic necrosis were both increased, particularly in cells with CA125 stimulation. Correspondingly, anti-MSLN dramatically reduced the number of viable cells.

\section{CA1 25 facilitated ovarian cancer metastasis in vivo}

Our previous studies demonstrated that CA125 significantly promoted cell migration in vitro [19]. To further confirm the role of CA125 in metastasis, we subcutaneously inoculated A2780 ovarian cancer cells expressing low CA125 levels into NOD-SCID-IL2rg mice. PBS or CA125 was injected via the tail vein once when the subcutaneous tumors were palpable (about $\left.20 \mathrm{~mm}^{3}\right)$. The tumor volumes were measured consecutively (Figure 7A), and the tumor images were taken after the mice were sacrificed (Figure 7B). Taken together, these results indicate that tumor volumes were not significantly different after CA125 injection compared with PBS injection. However, the inguinal and axillary lymphatic tissues were enlarged by CA125 injection (Figure 7C). Accordingly, tumor metastasis in the lymphatic vessels was observed with H\&E staining of the lymphatic tissues (Figure $7 \mathrm{D}$ ). The lymphatic vessels with tumor cells were recorded as positive lymphatic vessels, and normal lymphatic vessels were recorded as negative lymphatic vessels. The proportion of positive lymphatic vessels in the inguinal and axillary lymphatic tissues was determined (Figure 7E). The metastases were similar in the inguinal lymphatic tissues in both the CA125-stimulated and PBS groups. However, the proportion of positive lymphatic vessels in the axillary lymphatic tissues increased in the CA125-stimulated group, indicating that CA125 induced metastasis to axillary lymphatic tissues in vivo. One potential reason is that the tumor cells were injected near the hind limbs; thus, the cells were more likely to invade the inguinal lymphatic tissues. Figure $7 \mathrm{~F}$ is a typical histochemical staining picture of lymphatic vessel endothelial hyaluronan receptor-1 (LYVE-1). Lymphatic vessel endothelial cells in the lymphatic vessels were stained for LYVE-1.

\section{Anti-MSLN inhibited tumorigenesis and metastasis facilitated by CA 125}

Next, the therapeutic effects of anti-MSLN on ovarian cancer growth and metastasis were estimated in vivo. OVCAR3 cells expressing high levels of CA125 were chosen to evaluate the therapeutic effect. CA125 and PBS were injected in triplicate before four therapies followed, as the schematic diagram shows (Figure 8A). Similar to prior results, CA125 had no effect on tumor volume. However, anti-MSLN significantly reduced the tumor volume compared with that of the CA125 and PBS groups (Figure 8B-C). Accordingly, the lymphatic tissues were stripped, and the proportion of positive lymphatic vessels in the lymphatic tissues was determined according to $\mathrm{H} \& \mathrm{E}$ staining (Figure 8D-F). Figure 8G shown lymphatic vessel endothelial cells in the lymphatic vessels were stained for LYVE-1. The proportion of positive lymphatic vessels in the anti-MSLN group exhibited a downward trend. Evan's blue lymphangiogram was also used to evaluate tumor metastasis. The mesenteric tissues of mice injected with CA125 were hyperchromatic and were roughly eliminated when the mice were injected with anti-MSLN (Figure 8H). In brief, anti-MSLN treatment reduced tumorigenesis and metastasis.

\section{Discussion}

DKK1 is a secreted glycoprotein that shares a binding receptor with Wnt to LRP5/6 (low-density lipoprotein receptor-related protein 5/6) [24]. In the canonical Wnt pathway, DKK1 can block Wnt binding to the receptor and induce the release of Axin, therefore resulting in $\beta$-catenin degradation and translocation to the nucleus [25]. In adults, DKK1 is implicated in cancer. In prostate cancer, elevated DKK1 expression is an early event, but as cancer progresses, DKK1 expression declines, particularly in advanced bone metastases [26]. In breast cancer cells, DKK1 inhibits migration and invasion though suppression of the $\beta$-catenin/MMP7 pathway [27]. These findings indicate that DKK1 may be involved in cancer migration and metastasis. In epithelial ovarian cancer, research has revealed that ten-eleven translocation 1 (TET1) inhibits cell migration and invasion by upregulating DKK1 [28], which is partly consistent with our study. To dissect the effect of DKK1 in ovarian cancer, we analyzed several public databases. The DKK1 expression levels were downregulated in ovarian cancer tumors and correlated with FIGO stages, grades and disease-free survival. However, the serum CA125 level, as a tumor marker, was mostly elevated in ovarian cancer 
patients. Our data demonstrated that the DKK1 expression level was downregulated by elevated CA125 and was related to migration induced by CA125 in ovarian cancer cells in vitro. Traditionally, DKK1 is considered an inhibitor of the Wnt/ $\beta$-catenin

A

MA plot of Control VS CA125 stimulated group

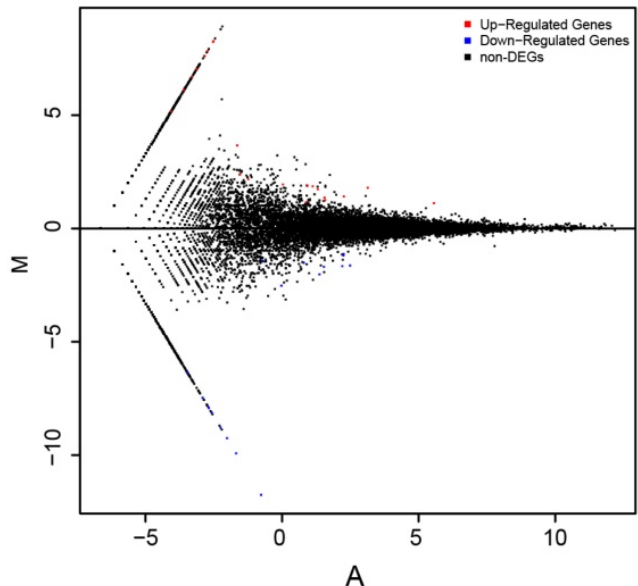

C

Hierarchical Clustering of Genes

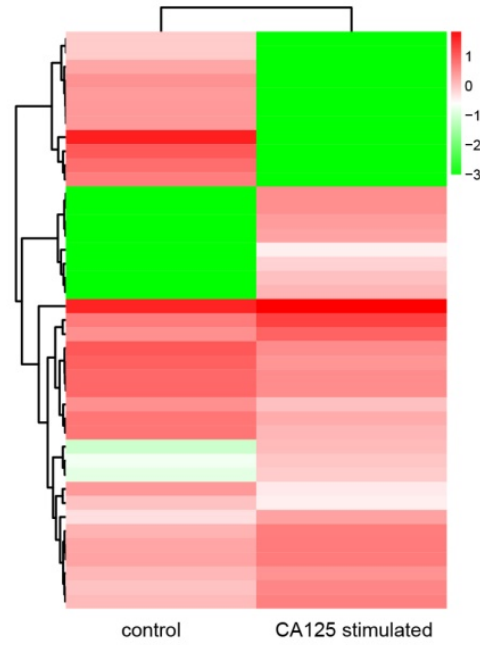

$\mathbf{E}$

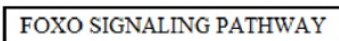

pathway. However, our data revealed that although CA125 strongly decreased the expression levels of DKK1, the Wnt/ $\beta$-catenin pathway was inactive in ovarian cancer cells.

\section{B}

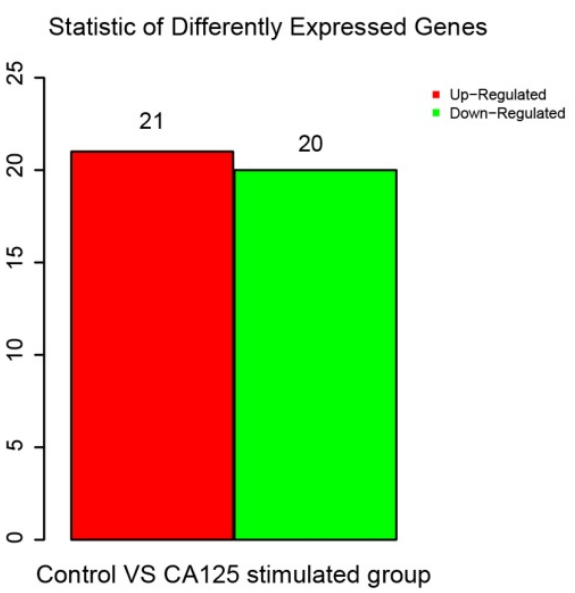

D

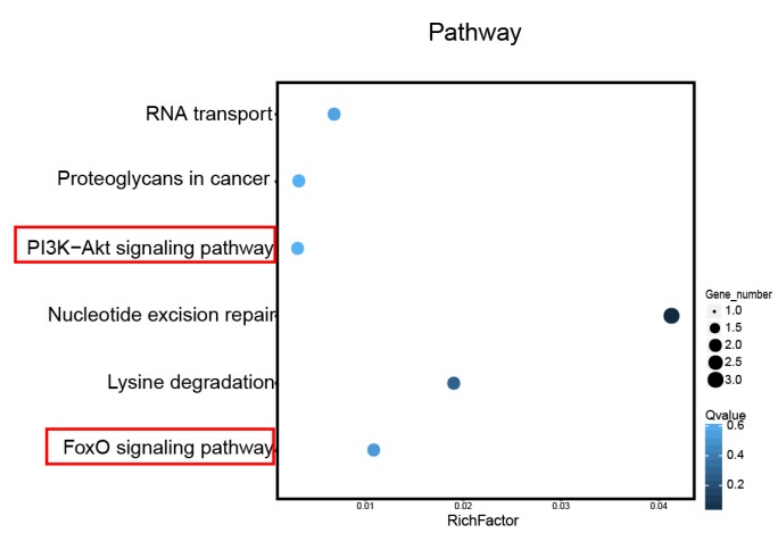

HYPOTHESIS

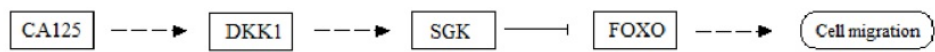

Figure 4. Next-generation sequencing of the differential expression of transcripts of ovarian cancer cells with CA125 stimulation. (A) Distribution of the differentially expressed transcripts in the MA plot. Red dots represent upregulated DEGs, blue dots represent downregulated DEGs, and black dots represent nondifferentially expressed genes. The abscissa is log2 (FPKM), and the ordinate log2 (FC). (B) Statistical histogram of DEGs. (C) Hierarchical clustering analysis of DEGs. (D) Enrichment scatter diagram of KEGG analyses of DEGs. The vertical axis represents the pathway, the horizontal axis represents the rich factor, the size of the point represents the number of DEGs in the pathway, and the color of the point corresponds to different Q-values. (E) Schematic diagram of the FOXO pathway and the hypothesis of the mechanism by which CA 125 enhances cell migration. 


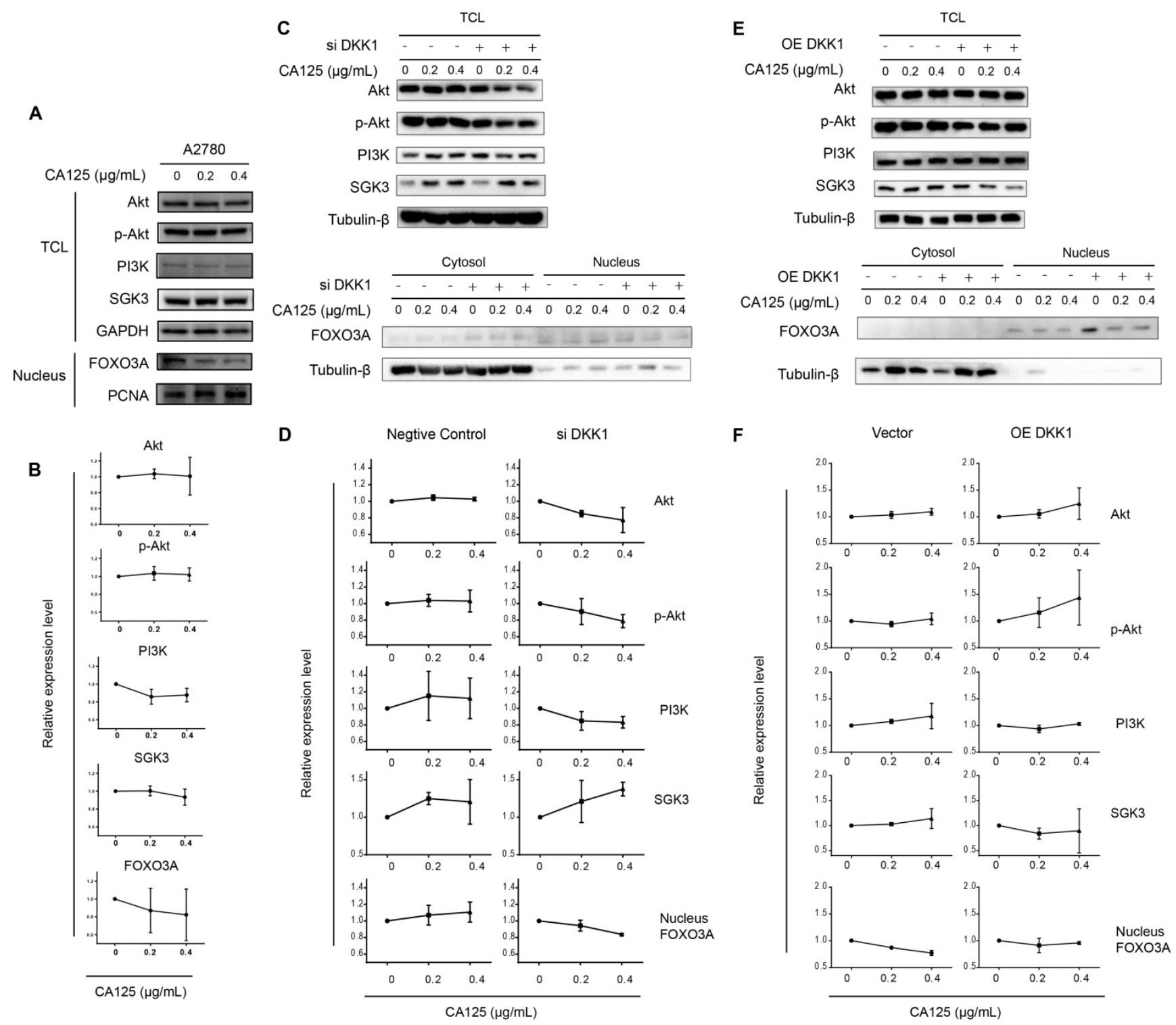

Figure 5. CA1 25 reduces DKK1 expression to activate the SGK3/FOXO3 pathway. (A) (C) (E) Western blots were performed to evaluate the expression of Akt, p-Akt, PI3K, and SGK3 in total cell lysates (TCL) and FOXO3 in cytoplasmic/nuclear protein extracts. GAPDH, Tubulin- $\beta$ and PCNA were used as the experimental controls. (B) (D) (F) Relative protein expression of blots was quantified using densitometric analysis. The results represent the mean \pm SD. There was no statistically significant difference.

In addition to the precise dysregulation of the Wnt signaling pathway, DKK1 is related to the PI3K/Akt pathway. In a recent study by Orestis Lyros et al., knocking down DKK1 significantly activated Akt phosphorylation independent of the Wnt pathway [29]. It has been demonstrated that DKK1 binds to cytoskeleton-associated protein 4 (CKAP4) with high affinity, leading to the activation of Akt by forming a complex between CKAP4 and PI3K, resulting in the proliferation of normal cells and cancer cells [30]. Our data support the theory that DKK1 is related to pathways other than the Wnt $/ \beta$-catenin pathway.

The serum- and glucocorticoid-regulated kinase (SGK) family, composed of three isoforms, is similar to Akt kinases, sharing similar structure, substrate specificity and function with Akt; both are activated downstream of the PI3K pathway [31,32]. Increasing studies have shown that SGK3 is dysregulated in cancer in an Akt-independent manner [33,34]. In prostate cancer, SGK3 is an androgen receptor transcriptional target and promotes cancer cell proliferation [35]. Forkhead box $\mathrm{O}$ (FOXO) transcription factor can regulate cells by modulating apoptosis [36], the cell cycle [37], DNA repair [38] and migration [39]. SGK3 recognizes the same substrate phosphorylation motif as Akt, RXRXXS/T (R indicates arginine, $X$ indicates any amino acid, and $\mathrm{S} / \mathrm{T}$ indicates serine/threonine), and phosphorylates FOXO3 in preferred sites [40]. Phosphorylated FOXO3 increases binding with 14-3-3 proteins. The binding prevents FOXO3 nuclear reimport, leading to the translocation of $\mathrm{FOXO} 3$ from the nucleus to the cytoplasm [42]. Our data suggest that CA125 activates 
the SGK3/FOXO3 pathway by reducing the expression of DKK1. The translocation from the nucleus to the cytosol of FOXO3 was observed under CA125 stimulation, which operated in synergy with siDKK1 and was reversed by OE DKK1. Notably, SGK3 and Akt share overlapping substrates that modulate feedback inhibition [45]. Akt and p-Akt were feedback inhibited under the joint effects of CA125 and siDKK1.

Mesothelin (MSLN) was first described in the early 1990s by Ira Pastan and Mark Willingham [46]. This protein is expressed on the surface of human ovarian carcinoma cells and normal mesothelium. [47]. MSLN was estimated to be strongly overexpressed in nearly one-third of human malignancies and is therefore a very important target for immunotherapy [49,50]. MSLN is highly expressed in $70 \%$ of ovarian cancers, including up to $100 \%$ of serous papillary ovarian cancers [51,52]. Initial experiments suggested that mesothelin is involved in cell adhesion events [48]. In 2004, researchers demonstrated the binding of MSLN to CA125 by flow cytometry as well as
A

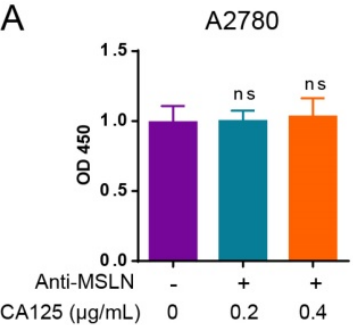

B

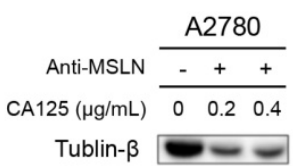

C
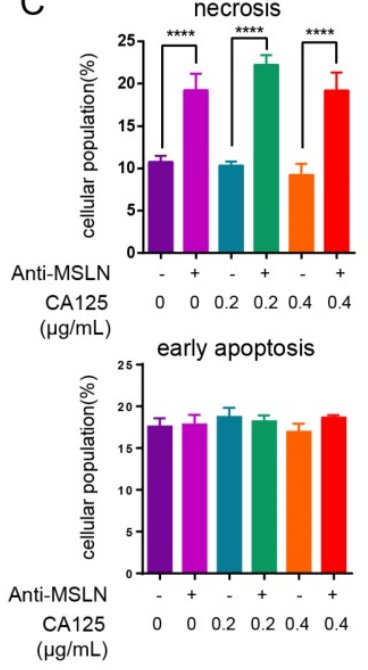
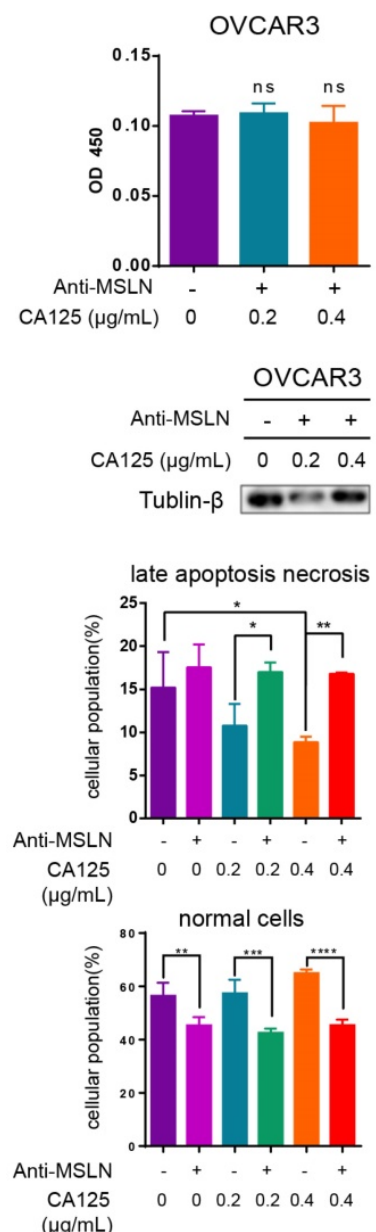

normal cells

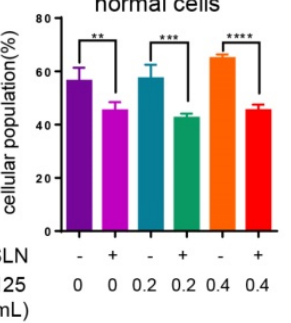

immunoprecipitation and found that CA125 and MSLN are co-expressed in advanced ovarian cancer. The binding of CA125 and membrane MSLN might contribute to the metastasis of ovarian cancer by initiating cell attachment [53]. Mesothelin-CA125 binding has been proven to be a high affinity, $\mathrm{N}$-glycan-dependent interaction that increases cell adhesion [54]. The binding occurs with a strong affinity with an apparent $\mathrm{Kd}$ of $5-10 \mathrm{nM}$, and the $\mathrm{N}$-linked oligosaccharides of CA125 and the $\mathrm{N}$-terminus of mesothelin are necessary in this binding [54,55]. A study proved that CA125 has different mesothelin-binding abilities in patients with endometriosis and epithelial ovarian cancer. This mesothelin-binding ability was significantly higher in epithelial ovarian cancer patients than in patients with endometriosis [57]. Thus, in OC patients, CA125 may have a strong affinity for binding to mesothelin in ovarian cancer cells to trigger further effects that enhance metastasis. Our data showed that anti-MSLN can block the reduction of DKK1 mediated by CA125 in ovarian cancer cells.

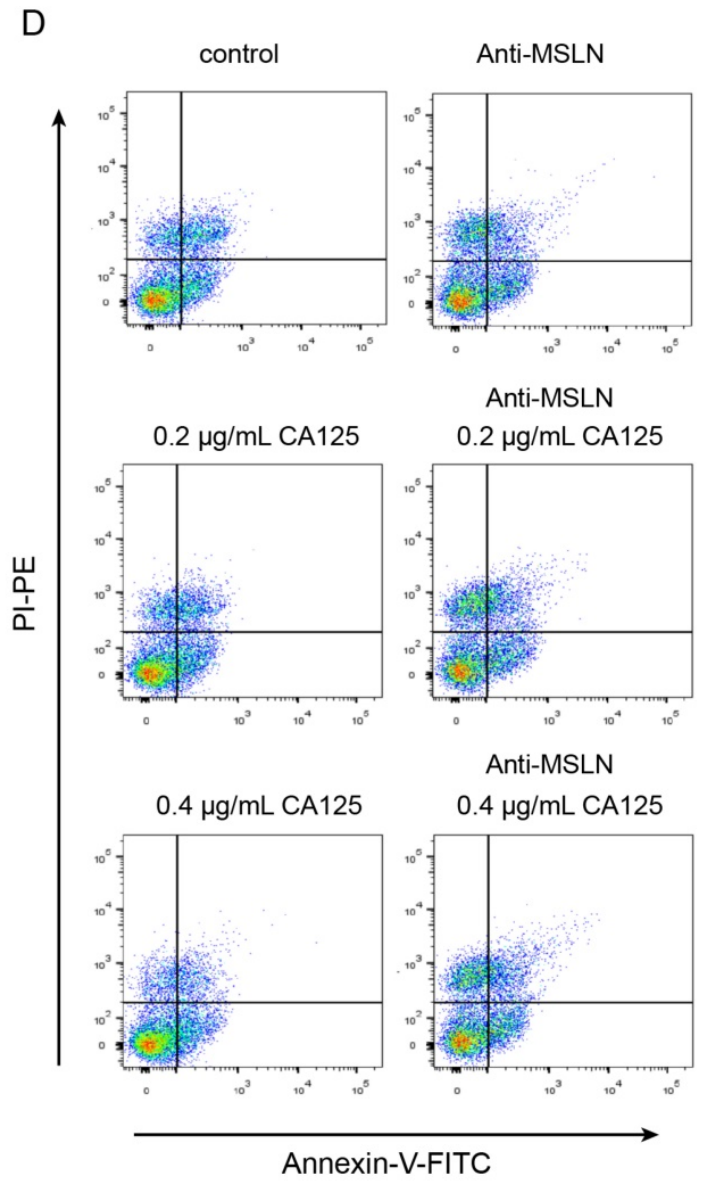

Figure 6. Anti-MSLN blockade of CA1 25 reduced the decrease in DKK1 and initiated apoptosis. (A) Expression of DKK1 was determined by ELISAs. (B) The expression levels of Tubulin- $\beta$ in total cell lysates were determined by Western blots. (C-D) Flow cytometric analysis of OVCAR3 cells treated with and without anti-MSLN or CA125. Statistical analysis of the gated cells and representative images are shown. The results represent the mean $\pm S D$. ${ }^{*} \mathrm{p}<0.05,{ }^{* *} \mathrm{p}<0.01,{ }^{* * *} \mathrm{p}<0.001,{ }^{*} * * * \mathrm{p}<0.0001, \mathrm{~ns}, \mathrm{not}$ significant. 
A

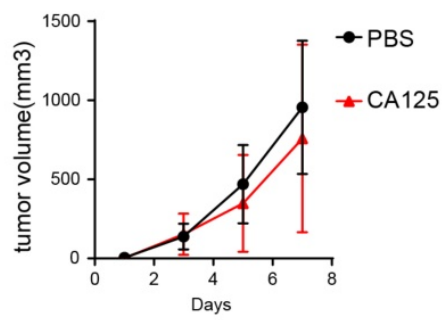

C

Inguinal lymphatic tissue

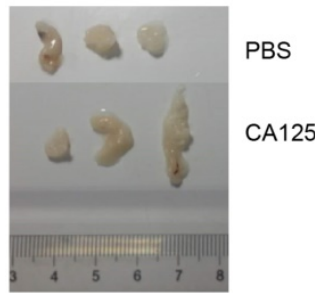

E

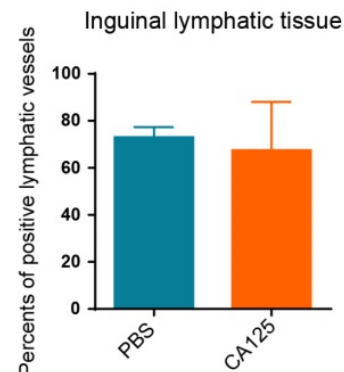

B

Tumors

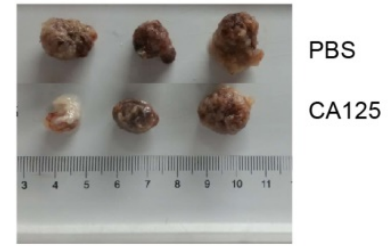

CA125

Axillary lymphatic tissue

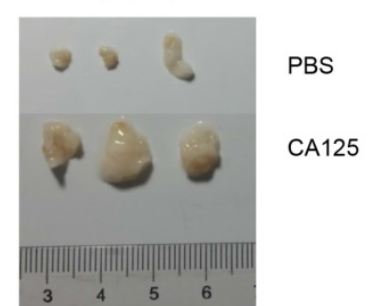

Axillary lymphatic tissue
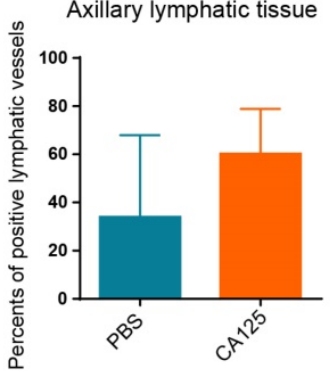

D

$\mathrm{F}$

CA125

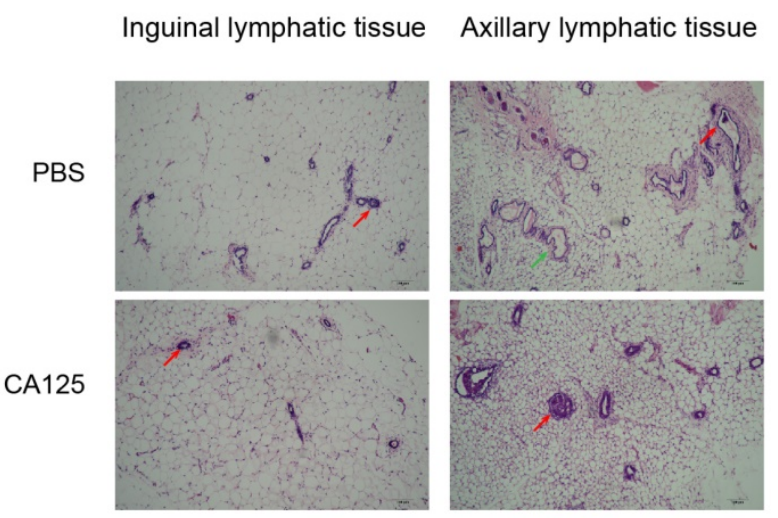
Inguinal lymphatic tissue Axillary lymphatic tissue
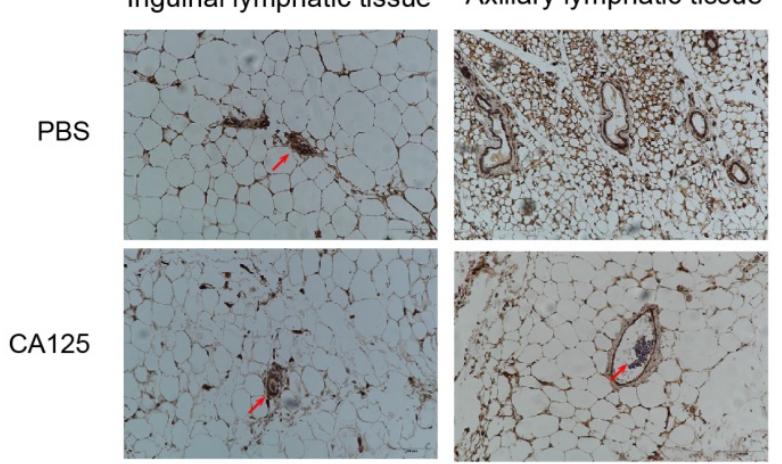

Figure 7. CA125 facilitates ovarian cancer metastasis in vivo. (A) The effect of CA125 on tumor growth was monitored by measuring tumor volume consecutively. Individual tumor images (B) and lymphatic tissue images (C) were taken after the mice were sacrificed. (D) Representative micrographs of hematoxylin and eosin (H\&E) staining (scale bars $=100 \mu \mathrm{m})$. Red arrows indicate metastases. Green arrows indicate normal lymphatic vessels. (E) The percentages of positive lymphatic vessels in the inguinal and axillary lymphatic tissues. The results represent the mean \pm SD. (F) LYVE-1 histochemical staining of inguinal and axillary lymphatic tissues. The red arrow shows the tumor cells in the lymphatic vessel (scale bars $=100 \mu \mathrm{m})$.

In addition, MSLN protects cells from drug-induced apoptosis and mediates drug resistance in cancer $[58,59,60]$. In our studies, anti-MSLN was proven to induce apoptosis and necrosis of ovarian cancer cells. The possible mechanism of apoptosis is as follows: anti-MSLN can block CA125 from binding with mesothelin, which reverses the effect of CA125 on cell migration and apoptosis, and anti-MSLN inhibits the ability of mesothelin to protect cells from apoptosis.

Schematic diagram (Figure 9) showing the potential mechanism of the anti-MSLN antibody blocking the effect of CA125 on inducing migration. High expression of CA125 on ovarian cancer cells results in release of the fragment free CA125, which binds to the mesothelin on the surface of ovarian cancer cells to reduce DKK1 expression. The reduction of DKK1 activated the SGK3/FOXO3 pathway to accelerate cell migration and tumor metastasis (Figure 9A). Once anti-MSLN was added, free-CA125 could no longer bind with ovarian cancer cells. The combination of anti-MSLN and ovarian cancer cells reversed migration and apoptosis (Figure 9B).

In tumor xenograft mice, CA125 was proven to promote the metastasis of ovarian cancer. Compared with those of the mice injected with PBS, the axillary lymphatic tissues of the mice injected with CA125 were enlarged, and the proportion of lymphatic vessels containing tumor cells was increased. These results suggested that CA125 can promote the metastasis of ovarian cancer in mice.

The therapeutic effects of anti-MSLN on ovarian cancer growth and metastasis were confirmed in vivo. The results showed that the tumors of mice injected with anti-MSLN were smaller than those injected with PBS or CA125, indicating that tumor growth was inhibited by anti-MSLN. At present, Evan's blue lymphatic tracer is often used in mice to assess tumor metastasis in mice [61]. In our experiment, mesenteric drainage was increased in the CA125-treated mice but decreased in the mice treated with anti-MSLN. These results indicated that anti-MSLN can inhibit the growth and metastasis of ovarian cancer. 
A

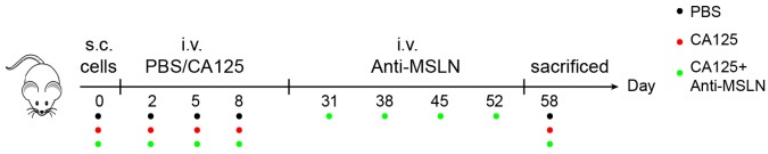

E

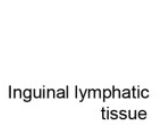

B

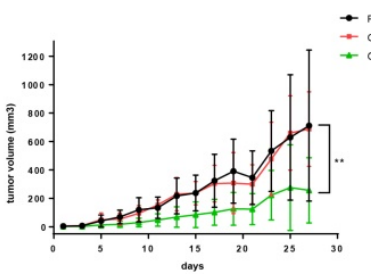

D

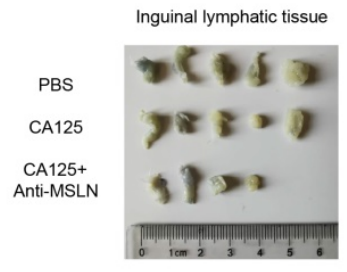

G

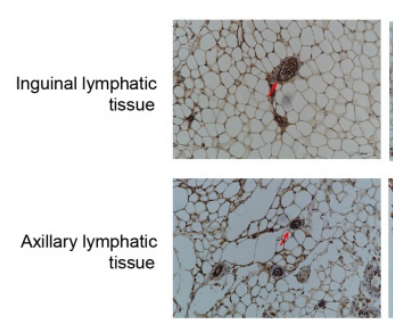

c C CA125 CA125+ Anti-is
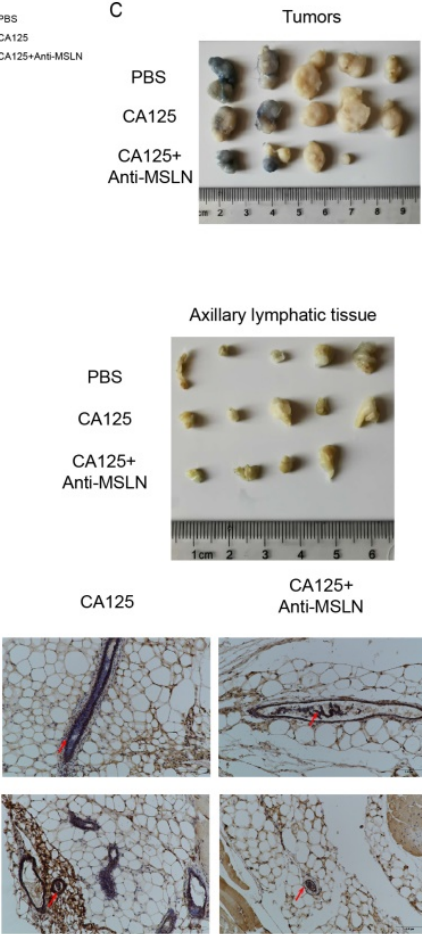

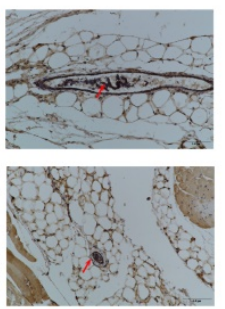

Axillary Iymphatic

F
PBS
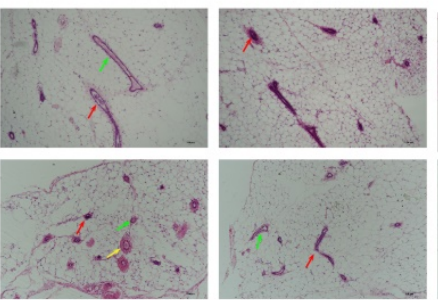

Anti-MSL
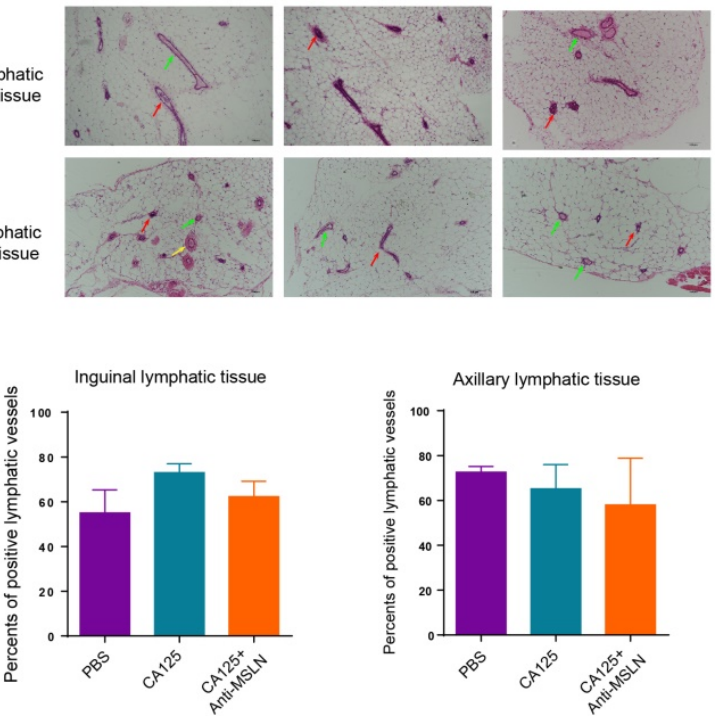

$\mathrm{H}$

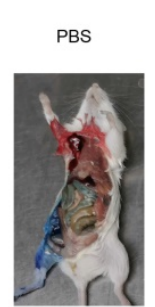

CA125

CA125+
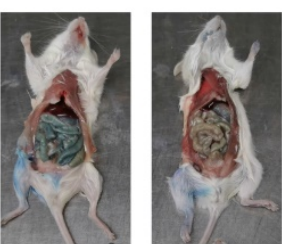

Figure 8. Anti-MSLN inhibited tumorigenesis and metastasis facilitated by CA125. (A) Schematic diagram of therapeutic strategies of anti-MSLN. (B) The therapeutic effect of anti-MSLN on tumor growth was monitored by consecutively measuring tumor volume. Individual tumor images (C) and lymphatic tissue images (D) were taken after the mice were sacrificed. (E) Representative micrographs of H\&E staining (scale bars $=100 \mu \mathrm{m}$ ). Red arrows indicate metastases. Green arrows indicate normal lymphatic vessels. Yellow arrows indicate the capillary. (F) Statistics of the percentages of positive lymphatic vessels in the inguinal and axillary lymphatic tissues. The results represent the mean \pm SD. (G) LYVE-1 histochemical staining of inguinal and axillary lymphatic tissues. The red arrow shows the tumor cells in the lymphatic vessel (scale bars $=100 \mu \mathrm{m}$ ). (H) Evan's blue lymphangiogram was used to evaluate tumor metastasis. Two-way ANOVA tests were used to compare the differences in tumor volume. The results represent the mean $\pm \mathrm{SD}$. ${ }^{* *} \mathrm{p}<0.01$.

A

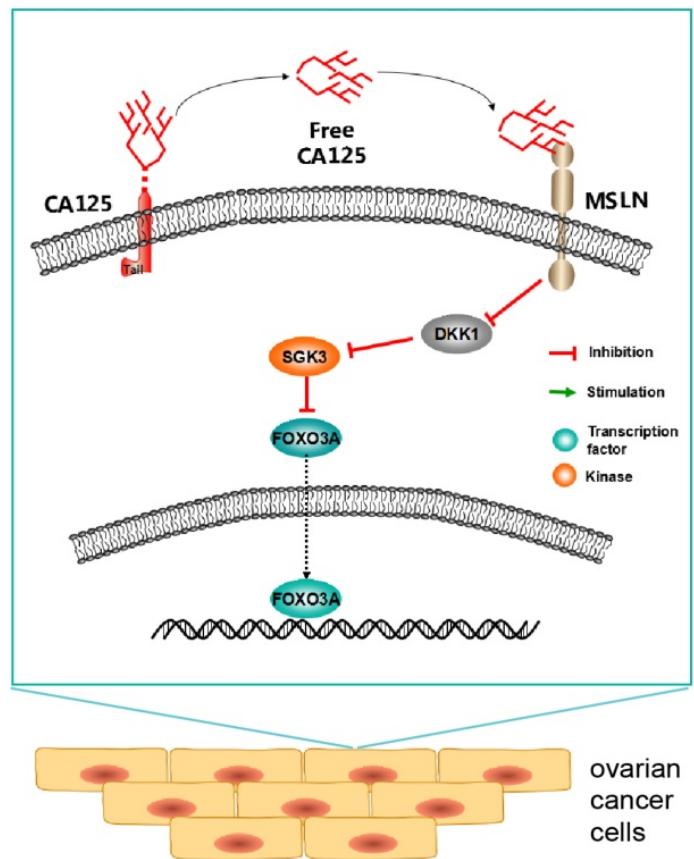

B

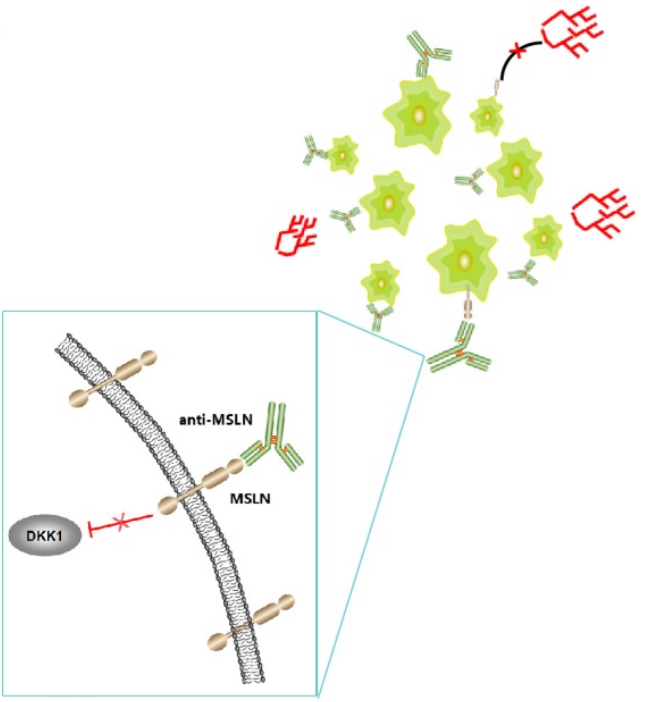

apoptosis of ovarian cancer cells

(4) free CA125

Anti-MSLN

ii MSLN

Figure 9. Schematic diagram of the study. (A) Schematic diagram of the potential mechanism of CA125-induced migration. (B) Schematic diagram of the potential therapeutic mechanism. 


\section{Conclusion}

In summary, the expression levels of DKK1 were downregulated in ovarian cancer patients and correlated with FIGO stage, grade and disease-free survival. Our data suggest that CA125 enhanced the migration and activated the SGK3/FOXO3 pathway by decreasing DKK1. Anti-MSLN can block the reduction of DKK1 mediated by CA125 and initiate apoptosis in vitro. In vivo, our data suggest that antibodies targeting mesothelin may offer a therapeutic option for ovarian cancer patients with high levels of serum CA125.

\section{Abbreviations}

OC: ovarian cancer; CA125: cancer antigen 125; MUC16: Mucin 16; DKK1: Dickkopf-1; SGK3: serum/glucocorticoid regulated kinase family member 3; FOXO3: forkhead box O3; MSLN: mesothelin; GAPDH: glyceraldehyde-3-phosphate dehydrogenase; PI3K: phosphatidylinositol-3 kinase; TET1: Ten-eleven translocation 1; LPR5/6: Low density lipoprotein receptor-related protein 5/6; CKAP4: cytoskeleton-associated protein 4; DEGs: differentially expressed genes; MPF: megakaryocyte potentiating factor; TNF: tumor necrosis factor; ELISA: enzyme-Linked Immuno Sorbent Assay; OD: optical density; q-PCR: Quantitative Polymerase chain reaction; SDS-PAGE: Sodium dodecyl sulfatePolyacrylamide gelelectrophoresis; PBS: Phosphatebuffered saline; siRNA: Small interfering RNA; OE: Over Expression; FC: fold change; FDR: False Discovery Rate; KEGG: Kyoto Encyclopedia of Genes and Genomes; FITC: fluorescein isothiocyanate; PI: Propidium Iodide; MW: molecular weight.

\section{Supplementary Material}

Supplementary figures and tables. http://www.ijbs.com/v17p0574s1.pdf

\section{Acknowledgements}

This work is supported by the scientific research projects of Tianjin Education Commission (2018KJ077) and the research funds of Tianjin Nankai Hospital (81971858-2).

We also thank Qin Yuan and You Cheng for their assistances to the experiments.

\section{Author Contributions}

Qianyu Huo analyzed and wrote the manuscript, Chen $\mathrm{Xu}$ validated the operation, Yunde Liu administrated and supervised the project, Huijing Bao designed the studies, Yanhong Shao, Qin $\mathrm{Yu}$ and Lunhui Huang reviewed and edited the manuscript.
All the authors read and approved the final manuscript.

\section{Competing Interests}

The authors have declared that no competing interest exists.

\section{References}

1. Muinao T, Pal M, Deka BH. Origins based clinical and molecular complexities of epithelial ovarian cancer. Int J Biol Macromol. 2018; 118: 1326-45.

2. Chien J, Poole EM. Ovarian Cancer Prevention, Screening, and Early Detection: Report From the 11th Biennial Ovarian Cancer Research Symposium. Int J Gynecol Cancer. 2017; 27: S20-2.

3. Reid BM, Permuth JB, Sellers TA. Epidemiology of ovarian cancer: a review. Cancer Biol Med. 2017; 14: 9-32.

4. Liang H, Zhao X, Wang C, Sun J, Chen Y, Wang G, et al. Systematic analyses reveal long non-coding RNA (PTAF)-mediated promotion of EMT and invasion-metastasis in serous ovarian cancer. Mol Cancer. 2018; 17: 96.

5. Prat J. Abridged republication of FIGO's staging classification for cancer of the ovary, fallopian tube, and peritoneum. Cancer-Am Cancer Soc. 2015; 121: 3452-4.

6. Morales-Vasquez F, Pedernera E, Reynaga-Obregon J, Lopez-Basave HN, Gomora MJ, Carlon E, et al. High levels of pretreatment CA125 are associated to improved survival in high grade serous ovarian carcinoma. J Ovarian Res. 2016; 9: 41.

7. Jelovac D, Armstrong DK. Recent progress in the diagnosis and treatment of ovarian cancer. CA Cancer J Clin. 2011; 61: 183-203.

8. Bon GG, Kenemans P, Verstraeten R, van Kamp GJ, Hilgers J. Serum tumor marker immunoassays in gynecologic oncology: establishment of reference values. Am J Obstet Gynecol. 1996; 174: 107-14.

9. Bast RI, Klug TL, St JE, Jenison E, Niloff JM, Lazarus $H$, et al A radioimmunoassay using a monoclonal antibody to monitor the course of epithelial ovarian cancer. N Engl J Med. 1983; 309: 883-7.

10. Guo N, Peng Z. Does serum CA125 have clinical value for follow-up monitoring of postoperative patients with epithelial ovarian cancer? Results of a 12-year study. J Ovarian Res. 2017; 10: 14.

11. Muallem MZ, Parashkevova A, Almuheimid J, Richter R, Diab Y, Braicu EI, et al. Preoperative CA-125 Values as a Predictive Factor for the Postoperative Outcome in Primary Serous Ovarian Cancer. Anticancer Res. 2017; 37: 3157-61.

12. Yin BW, Lloyd KO. Molecular cloning of the CA125 ovarian cancer antigen: identification as a new mucin, MUC16. J Biol Chem. 2001; 276: 27371-5.

13. Kasprzak A, Adamek A. Mucins: the Old, the New and the Promising Factors in Hepatobiliary Carcinogenesis. Int J Mol Sci. 2019; 20.

14. Desseyn JL, Tetaert D, Gouyer V. Architecture of the large membrane-bound mucins. Gene. 2008; 410: 215-22.

15. van Putten J, Strijbis K. Transmembrane Mucins: Signaling Receptors at the Intersection of Inflammation and Cancer. J Innate Immun. 2017; 9: 281-99.

16. Drescher $\mathrm{CW}$, Shah $\mathrm{C}$, Thorpe J, O'Briant $\mathrm{K}$, Anderson GL, Berg CD, et al. Longitudinal screening algorithm that incorporates change over time in CA125 levels identifies ovarian cancer earlier than a single-threshold rule. J Clin Oncol. 2013; 31: 387-92.

17. Felder M, Kapur A, Gonzalez-Bosquet J, Horibata S, Heintz J, Albrecht R, et al. MUC16 (CA125): tumor biomarker to cancer therapy, a work in progress. Mol Cancer. 2014; 13: 129

18. Theriault C, Pinard M, Comamala M, Migneault M, Beaudin J, Matte I, et al. MUC16 (CA125) regulates epithelial ovarian cancer cell growth, tumorigenesis and metastasis. Gynecol Oncol. 2011; 121: 434-43.

19. Yuan Q, Song J, Yang W, Wang H, Huo Q, Yang J, et al. The effect of CA125 on metastasis of ovarian cancer: old marker new function. Oncotarget. 2017; 8: 50015-22.

20. Tan TZ, Yang H, Ye J, Low J, Choolani M, Tan DS, et al. CSIOVDB: a microarray gene expression database of epithelial ovarian cancer subtype. Oncotarget. 2015; 6: 43843-52.

21. Huang H, Tindall DJ. Dynamic FoxO transcription factors. J Cell Sci. 2007; 120: 2479-87.

22. Avula LR, Rudloff M, El-Behaedi S, Arons D, Albalawy R, Chen X, et al. Mesothelin Enhances Tumor Vascularity in Newly Forming Pancreatic Peritoneal Metastases. Mol Cancer Res. 2020; 18: 229-39.

23. de Castro E, Reus TL de Aguiar AM, Avila AR, de Arruda CBDS Procaspase-activating compound-1 induces apoptosis in Trypanosoma cruzi. Apoptosis. 2017; 22: 1564-77.

24. Mao B, Wu W, Davidson G, Marhold J, Li M, Mechler BM, et al. Kremen proteins are Dickkopf receptors that regulate Wnt/beta-catenin signalling. Nature. 2002; 417: 664-7.

25. Zorn AM. Wnt signalling: antagonistic Dickkopfs. Curr Biol. 2001; 11: R592-5.

26. Hall CL, Daignault SD, Shah RB, Pienta KJ, Keller ET. Dickkopf-1 expression increases early in prostate cancer development and decreases during progression from primary tumor to metastasis. Prostate. 2008; 68: 1396-404. 
27. Niu J, Li XM, Wang X, Liang C, Zhang YD, Li HY, et al. DKK1 inhibits breast cancer cell migration and invasion through suppression of beta-catenin/MMP7 signaling pathway. Cancer Cell Int. 2019; 19: 168.

28. Duan H, Yan Z, Chen W, Wu Y, Han J, Guo H, et al. TET1 inhibits EMT of ovarian cancer cells through activating Wnt/beta-catenin signaling inhibitors DKK1 and SFRP2. Gynecol Oncol. 2017; 147: 408-17.

29. Lyros O, Lamprecht AK, Nie L, Thieme R, Gotzel K, Gasparri M, et al. Dickkopf-1 (DKK1) promotes tumor growth via Akt-phosphorylation and independently of Wnt-axis in Barrett's associated esophageal adenocarcinoma. Am J Cancer Res. 2019; 9: 330-46.

30. Kimura H, Fumoto K, Shojima K, Nojima S, Osugi Y, Tomihara H, et al. CKAP4 is a Dickkopf1 receptor and is involved in tumor progression. J Clin Invest. 2016; 126: 2689-705.

31. Basnet R, Gong GQ, Li C, Wang MW. Serum and glucocorticoid inducible protein kinases (SGKs): a potential target for cancer intervention. Acta Pharm Sin B. 2018; 8: 767-71.

32. Firestone GL, Giampaolo JR, O'Keeffe BA. Stimulus-dependent regulation of serum and glucocorticoid inducible protein kinase (SGK) transcription, subcellular localization and enzymatic activity. Cell Physiol Biochem. 2003; 13: 1-12.

33. Vasudevan KM, Barbie DA, Davies MA, Rabinovsky R, McNear CJ, Kim JJ, et al. AKT-independent signaling downstream of oncogenic PIK3CA mutations in human cancer. Cancer Cell. 2009; 16: 21-32.

34. Bruhn MA, Pearson RB, Hannan RD, Sheppard KE. Second AKT: the rise of SGK in cancer signalling. Growth Factors. 2010; 28: 394-408.

35. Wang Y, Zhou D, Chen S. SGK3 is an androgen-inducible kinase promoting prostate cancer cell proliferation through activation of p70 S6 kinase and up-regulation of cyclin D1. Mol Endocrinol. 2014; 28: 935-48.

36. Shukla S, Rizvi F, Raisuddin S, Kakkar P. FoxO proteins' nuclear retention and $\mathrm{BH} 3$-only protein Bim induction evoke mitochondrial dysfunction-mediated apoptosis in berberine-treated HepG2 cells. Free Radic Biol Med. 2014; 76: 185-99.

37. Kloet DE, Polderman PE, Eijkelenboom A, Smits LM, van Triest MH, van den Berg MC, et al. FOXO target gene CTDSP2 regulates cell cycle progression through Ras and p21(Cip1/Waf1). Biochem J. 2015; 469: 289-98.

38. Bianco JN, Schumacher B. MPK-1/ERK pathway regulates DNA damage response during development through DAF-16/FOXO. Nucleic Acids Res. 2018; 46: 6129-39.

39. Chen QB, Li ZH, Fu Y, Lv NN, Tian N, Han L, et al. Downregulated long non-coding RNA LINC00899 inhibits invasion and migration of spinal ependymoma cells via RBL2-dependent FoxO pathway. Cell Cycle. 2019; 18: 2566-79.

40. Boccitto M, Kalb RG. Regulation of Foxo-dependent transcription by post-translational modifications. Curr Drug Targets. 2011; 12: 1303-10.

41. Brunet A, Park J, Tran H, Hu LS, Hemmings BA, Greenberg ME. Protein kinase SGK mediates survival signals by phosphorylating the forkhead transcription factor FKHRL1 (FOXO3a). Mol Cell Biol. 2001; 21: 952-65.

42. Brunet A, Kanai F, Stehn J, Xu J, Sarbassova D, Frangioni JV, et al. 14-3-3 transits to the nucleus and participates in dynamic nucleocytoplasmic transport. J Cell Biol. 2002; 156: 817-28.

43. Brunet A, Bonni A, Zigmond MJ, Lin MZ, Juo P, Hu LS, et al. Akt promotes cell survival by phosphorylating and inhibiting a Forkhead transcription factor. Cell. 1999; 96: 857-68.

44. Zou Y, Tsai WB, Cheng CJ, Hsu C, Chung YM, Li PC, et al. Forkhead box transcription factor $\mathrm{FOXO3a}$ suppresses estrogen-dependent breast cancer cell proliferation and tumorigenesis. Breast Cancer Res. 2008; 10: R21.

45. Gasser JA, Inuzuka H, Lau AW, Wei W, Beroukhim R, Toker A. SGK3 mediates INPP4B-dependent PI3K signaling in breast cancer. Mol Cell. 2014; 56: 595-607.

46. Chang K, Pastan I, Willingham MC. Isolation and characterization of a monoclonal antibody, $\mathrm{K} 1$, reactive with ovarian cancers and normal mesothelium. Int J Cancer. 1992; 50: 373-81.

47. Pastan I, Hassan R. Discovery of mesothelin and exploiting it as a target for immunotherapy. Cancer Res. 2014; 74: 2907-12.

48. Chang K, Pastan I. Molecular cloning of mesothelin, a differentiation antigen present on mesothelium, mesotheliomas, and ovarian cancers. Proc Natl Acad Sci U S A. 1996; 93: 136-40.

49. Ren YR, Patel K, Paun BC, Kern SE. Structural analysis of the cancer-specific promoter in mesothelin and in other genes overexpressed in cancers. J Biol Chem. 2011; 286: 11960-9.

50. Ren YR, Patel K, Paun BC, Kern SE. Structural analysis of the cancer-specific promoter in mesothelin and in other genes overexpressed in cancers. J Biol Chem. 2011; 286: 11960-9.

51. Hassan R, Kreitman RJ, Pastan I, Willingham MC. Localization of mesothelin in epithelial ovarian cancer. Appl Immunohistochem Mol Morphol. 2005; 13: 243-7.

52. Muminova ZE, Strong TV, Shaw DR. Characterization of human mesothelin transcripts in ovarian and pancreatic cancer. Bmc Cancer. 2004; 4: 19

53. Rump A, Morikawa Y, Tanaka M, Minami S, Umesaki N, Takeuchi M, et al. Binding of ovarian cancer antigen CA125/MUC16 to mesothelin mediates cell adhesion. J Biol Chem. 2004; 279: 9190-8.

54. Gubbels JA, Belisle J, Onda M, Rancourt C, Migneault M, Ho M, et al. Mesothelin-MUC16 binding is a high affinity, $\mathrm{N}$-glycan dependent interaction that facilitates peritoneal metastasis of ovarian tumors. Mol Cancer. 2006; 5: 50.
55. Kaneko O, Gong L, Zhang J, Hansen JK, Hassan R, Lee B, et al. A binding domain on mesothelin for CA125/MUC16. J Biol Chem. 2009; 284: 3739-49.

56. Chen SH, Hung WC, Wang P, Paul C, Konstantopoulos K. Mesothelin binding to CA125/MUC16 promotes pancreatic cancer cell motility and invasion via MMP-7 activation. Sci Rep. 2013; 3: 1870.

57. Sasaki A, Akita K, Ito F, Mori T, Kitawaki J, Nakada H. Difference in mesothelin-binding ability of serum CA125 between patients with endometriosis and epithelial ovarian cancer. Int J Cancer. 2015; 136: 1985-90.

58. Chang MC, Chen CA, Hsieh CY, Lee CN, Su YN, Hu YH, et al. Mesothelin inhibits paclitaxel-induced apoptosis through the PI3K pathway. Biochem J. 2009; 424: 449-58.

59. Uehara N, Matsuoka Y, Tsubura A. Mesothelin promotes anchorage-independent growth and prevents anoikis via extracellular signal-regulated kinase signaling pathway in human breast cancer cells. Mol Cancer Res. 2008; 6: 186-93.

60. Bharadwaj U, Marin-Muller C, Li M, Chen C, Yao Q. Mesothelin confers pancreatic cancer cell resistance to TNF-alpha-induced apoptosis through Akt/PI3K/NF-kappaB activation and IL-6/Mcl-1 overexpression. Mol Cancer. 2011; 10: 106.

61. Das S, Sarrou E, Podgrabinska S, Cassella M, Mungamuri SK, Feirt N, et al. Tumor cell entry into the lymph node is controlled by CCL1 chemokine expressed by lymph node lymphatic sinuses. J Exp Med. 2013; 210: 1509-28. 